\title{
Comparação da Retração Aguda do Stent Entre o Suporte Vascular Bioabsorvível Eluidor de Everolimus e Dois Diferentes Stents Metálicos Farmacológicos
}

\author{
Tarcisio Campostrini Borghi Jr. ${ }^{1}$, J. Ribamar Costa Jr. ${ }^{2}$, Alexandre Abizaid ${ }^{3}$, Daniel Chamié ${ }^{4}$, \\ Mateus Veloso e Silva ${ }^{5}$, Danillo Taiguara ${ }^{6}$, Ricardo Costa ${ }^{7}$, Rodolfo Staico ${ }^{8}$, Fausto Feres ${ }^{9}$, \\ Áurea J. Chaves ${ }^{10}$, Dimytri Siqueira ${ }^{11}$, Amanda G. M. R. Sousa ${ }^{12}$, J. Eduardo Sousa ${ }^{13}$
}

\section{RESUMO}

Introdução: Suportes vasculares bioabsorvíveis (SVB) têm sido desenvolvidos como forma de fornecer sustentação à parede do vaso enquanto ocorre o processo de cicatrização, após a intervenção coronária percutânea (ICP), sendo absorvido posteriormente. Pelo fato da plataforma ser de material polimérico, existe preocupação em relação à retração aguda do dispositivo. Avaliamos aqui a retração aguda do SVB com a de dois diferentes stents farmacológicos metálicos. Métodos: Foram incluídos 50 pacientes com lesões não complexas. Dentre esses pacientes, 25 foram tratados com SVB e comparados a outros 25 pacientes tratados com stent de cromo-cobalto eluidor de everolimus ( $E E S ; n=12$ ) ou stent de aço inoxidável eluidor de biolimus (BES; $n=13$ ). A retração aguda foi definida como a diferença entre o diâmetro médio do balão durante a pressão máxima de inflação $(X)$ e o diâmetro médio do stent após o esvaziamento do balão $(\mathrm{Y})$. A porcentagem de retração aguda foi definida como $(X-Y) / X$. Resultados: Não houve diferença significativa em relação às características clínicas e angiográficas basais. O ganho luminal agudo foi menor com o SVB comparado ao EES e ao BES $(1,51 \pm 0,41 \mathrm{~mm}$ vs. $1,76 \pm 0,28 \mathrm{~mm}$ vs. $1,9 \pm 0,42 \mathrm{~mm} ; P=0,02)$. A retração aguda foi de 0,21 $\pm 0,13 \mathrm{~mm}$ vs. 0,15 $\pm 0,08 \mathrm{~mm}$ vs. $0,14 \pm 0,08 \mathrm{~mm}(\mathrm{P}=0,21)$, e o porcentual de retração aguda foi de $7,0 \pm 4,6 \%$ vs. $5,0 \pm 2,2 \%$ vs. $5,7 \pm 4,1 \%$ $(P=0,16)$. Conclusões: $O S V B$ demonstrou ter retração aguda

\section{ABSTRACT}

Comparison of Acute Stent Recoil Between the Everolimus-Eluting Bioresorbable Vascular Scaffold and Two Different Drug-Eluting Metallic Stents

Background: Bioresorbable vascular scaffolds (BVS) have been developed to provide support to the vessel wall during the healing process after percutaneous coronary intervention $(\mathrm{PCl})$, being reabsorbed afterwards. Because the scaffold is made of polymeric material, there is a concern regarding the acute recoil of the device. We compared the BVS acute recoil with that of two different metallic drug-eluting stents. Methods: Fifty patients with non-complex lesions were included. Twenty-five of these patients were treated with a BVS who were compared to 25 patients treated with a cobalt-chromium everolimus-eluting stent (EES, $n=12$ ) or a stainless steel biolimus-eluting stent (BES, $\mathrm{n}=13$ ). Acute recoil was defined as the difference between the mean diameter of the balloon during maximum inflation pressure $(X)$ and the mean diameter of the stent immediately after balloon deflation (Y). The percentage of acute recoil was defined as $(X-Y) / X$. Results: There was no significant difference in the baseline clinical and angiographic characteristics. Acute luminal gain was lower with BVS compared to EES and BES $(1.51 \pm 0.41 \mathrm{~mm}$ vs. $1.76 \pm 0.28 \mathrm{~mm}$ vs. $1.9 \pm 0.42 \mathrm{~mm}$, $\mathrm{P}=0.02$ ). Acute recoil was $0.21 \pm 0.13 \mathrm{~mm}$ vs. $0.15 \pm 0.08 \mathrm{~mm}$ vs. $0.14 \pm 0.08 \mathrm{~mm}(\mathrm{P}=0.21)$ and the percentage of acute

\footnotetext{
Residente do Serviço de Cardiologia Invasiva do Instituto Dante Pazzanese de Cardiologia. São Paulo, SP, Brasil.

2 Doutor. Cardiologista intervencionista do Serviço de Cardiologia Invasiva do Instituto Dante Pazzanese de Cardiologia. São Paulo, SP, Brasil. ${ }^{3}$ Livre-docente. Diretor da Divisão de Cardiologia Invasiva do Instituto Dante Pazzanese de Cardiologia. São Paulo, SP, Brasil.

${ }^{4}$ Cardiologista intervencionista do Serviço de Cardiologia Invasiva do Instituto Dante Pazzanese de Cardiologia. São Paulo, SP, Brasil.

5 Residente do Serviço de Cardiologia Invasiva do Instituto Dante Pazzanese de Cardiologia. São Paulo, SP, Brasil.

${ }^{6}$ Residente do Serviço de Cardiologia Invasiva do Instituto Dante Pazzanese de Cardiologia. São Paulo, SP, Brasil.

7 Doutor. Cardiologista intervencionista do Serviço de Cardiologia Invasiva do Instituto Dante Pazzanese de Cardiologia. São Paulo, SP, Brasil.

${ }^{8}$ Doutor. Cardiologista intervencionista do Serviço de Cardiologia Invasiva do Instituto Dante Pazzanese de Cardiologia. São Paulo, SP, Brasil.
}

\footnotetext{
${ }^{9}$ Doutor. Cardiologista intervencionista do Serviço de Cardiologia Invasiva do Instituto Dante Pazzanese de Cardiologia. São Paulo, SP, Brasil.

${ }^{10}$ Doutora. Cardiologista do Serviço de Cardiologia Invasiva do Instituto Dante Pazzanese de Cardiologia. São Paulo, SP, Brasil.

${ }^{11}$ Doutor. Cardiologista intervencionista do Serviço de Cardiologia Invasiva do Instituto Dante Pazzanese de Cardiologia. São Paulo, SP, Brasil. 12 Livre-docente. Diretora Geral do Instituto Dante Pazzanese de Cardiologia. São Paulo, SP, Brasil.

${ }^{13}$ Livre-docente. Diretor do Centro de Intervenções em Doenças Estruturais do Coração do Instituto Dante Pazzanese de Cardiologia. São
} Paulo, SP, Brasil.

Correspondência: Tarcisio Campostrini Borghi Jr. Avenida Dr. Dante Pazzanese, 500 - Vila Mariana - São Paulo, SP, Brasil - CEP 04012-180 E-mail: tarcisiocampostrini@gmail.com

Recebido em: 16/9/2012 • Aceito em: 10/11/2013 
ligeiramente maior, embora não significativa, que os stents metálicos farmacológicos de segunda geração.

DESCRITORES: Intervenção coronária percutânea. Stents farmacológicos. Implantes absorvíveis. Angiografia coronária.

$S$ tents metálicos farmacológicos são os dispositivos de escolha para o tratamento percutâneo da doença arterial coronária (DAC). Eles fornecem suporte à parede das artérias, prevenindo a retração aguda e tardia do vaso, além de inibirem a proliferação intimal intra-stent. Como consequência, o uso desses dispositivos alavancou as taxas de sucesso do procedimento e a durabilidade dos resultados. ${ }^{1}$

Entretanto, a permanência definitiva das hastes metálicas dos stents, com o consequente encarceramento do vaso, pode ocasionar alterações da motricidade e do remodelamento (fenômeno de Glagov), levando à má aposição das hastes e a alterações da conformabilidade do vaso. ${ }^{2}$

Na última década, a ideia de um suporte vascular transitório, que, por um determinado período, modulasse a hiperplasia intimal reparadora e, ao mesmo tempo, evitasse o remodelamento da artéria tratada, sendo, então, reabsorvido, tem ganhado destaque dentro da abordagem percutânea da doença coronária. Os suportes vasculares bioabsorvíveis (SVB), também comumente denominados "stents bioabsorvíveis", seriam os dispositivos que preencheriam esses requisitos. Entretanto, uma das principais dificuldades em desenvolver tais dispositivos, principalmente aqueles de composição polimérica, seria conferir força radial suficiente para impedir a ocorrência da retração aguda e tardia do vaso, não infrequente nos primeiros protótipos. ${ }^{3}$

Dentre os programas clínicos de SVB mais desenvolvidos, destaca-se o ABSORB $^{\circledR}$ (Abbott Vascular, Santa Clara, Estados Unidos), que combina uma plataforma polimérica e o fármaco antiproliferativo everolimus. Nesse estudo, objetivamos comparar a retração aguda do SVB ABSORB ${ }^{\circledR}$ Com a observada com stents farmacológicos metálicos de cromo-cobalto (Xience ${ }^{\circledR} \mathrm{V}$, Abbott Vascular, Santa Clara, Estados Unidos) e aço inoxidável (BioMatrix ${ }^{\oplus}$, Biosensors International, Singapura).

\section{MÉTODOS}

\section{Desenho do estudo e população-alvo}

Trata-se de um estudo retrospectivo, unicêntrico, desenvolvido no Serviço de Cardiologia Invasiva do Instituto Dante Pazzanese, em São Paulo (SP). A população deste estudo foi constituída pelos pacientes tratados com o SVB ABSORB $^{\circledR}$, BioMatrix ${ }^{\circledR}$ e Xience $V^{\circledR}$, recoil was $7.0 \pm 4.6 \%$ vs. $5.0 \pm 2.2 \%$ vs. $5.7 \pm 4.1 \%(P=0.16)$. Conclusions: BVS presented a slightly higher, although not significant, acute recoil than the two second-generation metallic drug-eluting stents.

DESCRIPTORS: Percutaneous coronary intervention. Durg-eluting stents. Absorbable implants. Coronary angiography.

como parte de protocolos locais desenvolvidos na referida instituição. Alguns critérios de inclusão foram adotados na presente análise, a fim de aproximar as coortes e minimizar possíveis vieses. Foram incluídos apenas pacientes com lesões únicas entre 70 e 90\% (determinação visual), do tipo de novo, em coronárias nativas de diâmetro entre 2,5 e 3,5 mm. A extensão máxima da lesão permitida foi de $23 \mathrm{~mm}$. Como característica comum a todo grupo, foram incluídos apenas pacientes tratados de maneira eletiva, não sendo permitida a inclusão de lesão-alvo em tronco de coronária esquerda, lesões ostiais, lesões com trombos, lesões com calcificação excessiva que exigisse técnicas atero-ablativas antes do implante do dispositivo e lesões em bifurcações com ramo lateral $>2 \mathrm{~mm}$.

\section{Dispositivos empregados}

O SVB ABSORB $^{\circledR}$ tem plataforma composta por polímero de ácido poli-L-láctico (PLLA), fármaco antiproliferativo everolimus e um sistema de entrega. $O$ corpo do SVB é revestido pela matriz de ácido poli-D, L-láctico (PDLLA) e everolimus, em uma relação 1:1. PLLA e PDLLA são totalmente metabolizados e absorvidos pelo organismo.

O stent Xience $^{\circledR} \vee$ é composto pela plataforma expansível por balão MULTI-LINK VISION ${ }^{\circledR}$ Com anéis em serpentina ligados por elos e fabricados a partir de uma única peça de cromo-cobalto, revestido com polímero durável contendo everolimus. A espessura das hastes é 0,081 $\mu \mathrm{m}$.

O stent BioMatrix ${ }^{\circledR}$ incorpora a plataforma S-Stent, um stent tubular de aço inoxidável, cortado a laser, com hastes de $112 \mu \mathrm{m}$. O fármaco antiproliferativo é o biolimus A9, um análogo do sirolimus altamente lipofílico, semissintético. Com base em estudos in vivo, o polímero de ácido poliláctico (PLA) biodegradável é totalmente convertido em ácido láctico em 6 a 9 meses.

\section{Procedimento}

Todos os procedimentos foram realizados de forma eletiva, de acordo com as diretrizes atuais. As lesões foram tratadas com técnicas habituais de intervenção, que incluíam pré-dilatação obrigatória com balão mais curto e com diâmetro 0,5 mm menor do que o dispositivo utilizado. A pós-dilatação, quando realizada (a critério do operador), deveria ser feita com balões 
não complacentes pelo menos $30 \%$ mais curtos que o SVB ou stent implantado.

A terapia antiplaquetária dupla pré-procedimento foi constituída por aspirina 100 a 200 mg/dia e dose de ataque de clopidogrel $300 \mathrm{mg}$, pelo menos 24 horas antes do procedimento, ou $600 \mathrm{mg}$ se $<24$ horas. Após a intervenção, aspirina 100 a 200 mg/dia foi prescrita indefinidamente e clopidogrel $75 \mathrm{mg} /$ dia foi mantido por um mínimo de 6 meses. Durante a intervenção coronária percutânea (ICP), a terapia antitrombínica consistiu de heparina não fracionada na dose de $100 \mathrm{UI} / \mathrm{kg}$ (ou $70 \mathrm{UI} / \mathrm{kg}$, em caso do uso de inibidor da glicoproteína IIb/IIla), objetivando-se alcançar tempo de coagulação ativado > 250 segundos (ou entre 200 e 250 segundos, no caso de uso de inibidor da glicoproteína IIb/IIla).

\section{Análise angiográfica coronária quantitativa}

As angiografias pré e pós-procedimento foram obtidas após a administração intracoronária de nitroglicerina (50 a $200 \mathrm{mg}$ ), em pelo menos duas projeções ortogonais correspondentes, sendo armazenadas em um formato DICOM para análise digital off-line. A análise angiográfica coronária quantitativa (QCA) foi realizada com um programa de computador dedicado, com detecção semiautomática das bordas do lúmen (QAngio XA versão 7.3, Medis Medical Imaging System, Leiden, Holanda). Análises angiográficas qualitativa e quantitativa foram realizadas por dois operadores experientes, seguindo protocolo predefinido. A ponta do cateter-guia cheio de contraste foi utilizada para a calibração. O diâmetro mínimo do lúmen (DML) e o diâmetro de referência (DR), obtidos por interpolação, foram usados para calcular o diâmetro da estenose: $\mathrm{DS}=(1-\mathrm{DML} / \mathrm{DR}) \times 100$. A retração aguda foi definida como a diferença entre o diâmetro médio do balão, durante a pressão máxima de inflação $(X)$, e o diâmetro luminal médio intra-stent, imediatamente após o esvaziamento do balão (Y); a percentagem da retração aguda foi definida como $(X-Y) / X$ (Figura).

\section{Análise estatística}

As variáveis categóricas foram apresentadas como frequências e porcentagens, sendo comparadas por meio do teste de análise de variância (ANOVA). Variáveis contínuas foram apresentadas como média e desvio padrão, sendo comparadas por meio do teste não paramétrico de Kruskal-Wallis. $O$ valor de $P<0,05$ foi considerado estatisticamente significativo. A análise estatística foi realizada utilizando o programa Statistical Package for the Social Sciences (SPSS), versão 19 para Windows (Chicago IL).

\section{RESULTADOS}

\section{Características clínicas}

Foram avaliados 50 pacientes submetidos à angioplastia eletiva, sendo 25 tratados com SVB, 12 tratados com EES e outros 13 com BES. As características clínicas estão resumidas na Tabela 1. Não houve diferença significativa entre as variáveis clínicas analisadas, exceto pela maior prevalência de infarto do miocárdio prévio nos grupos tratados com stents metálicos $(P=0,01)$.

\section{Variáveis angiográficas e do procedimento}

As variáveis angiográficas pré e pós-intervenção estão resumidas na Tabela 2 . O vaso mais abordado no grupo tratado com SVA foi a artéria descendente anterior, enquanto nos pacientes tratados com stents metálicos foi a artéria circunflexa $(P=0,24)$. Não houve diferença significante entre os grupos em relação ao DR

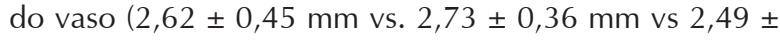
$0,44 \mathrm{~mm} ; \mathrm{P}=0,34)$ ou ao comprimento das lesões $(11,7 \pm 4,0 \mathrm{~mm}$ vs. $10,1 \pm 3,4 \mathrm{~mm}$ vs. $12,9 \pm 5,9 \mathrm{~mm}$; $\mathrm{P}=0,31)$. $\mathrm{O} \mathrm{DML}$ pré-procedimento não foi diferente entre os grupos $(0,87 \pm 0,32 \mathrm{~mm}$ vs. $0,90 \pm 0,25 \mathrm{~mm}$ vs. $0,78 \pm 0,44 \mathrm{~mm} ; \mathrm{P}=0,52$ ); após o procedimento, os pacientes tratados com o SVB apresentaram menor DML $(2,39 \pm 0,31 \mathrm{~mm}$ vs. 2,66 $\pm 0,26 \mathrm{~mm}$ vs. 2,69 \pm $0,45 \mathrm{~mm} ; \mathrm{P}<0,03)$. O ganho agudo foi menor com os SVB $(1,51 \pm 0,41 \mathrm{~mm}$ vs. 1,76 $\pm 0,28 \mathrm{~mm}$ vs. 1,9 \pm

TABELA 1

Características clínicas

\begin{tabular}{|c|c|c|c|c|}
\hline & $\begin{array}{c}\text { SVB } \\
(n=25)\end{array}$ & $\begin{array}{c}\text { EES } \\
(n=12)\end{array}$ & $\begin{array}{c}\text { BES } \\
(n=13)\end{array}$ & Valor de $\mathbf{P}$ \\
\hline Idade, anos & $56,8 \pm 7,0$ & $59,8 \pm 10,3$ & $61,0 \pm 4,8$ & 0,33 \\
\hline Sexo masculino, n (\%) & $15(60)$ & $6(50)$ & $9(69,2)$ & 0,39 \\
\hline Diabetes, n (\%) & $5(20)$ & 0 & $1(57,7)$ & 0,28 \\
\hline Hipertensão arterial, n (\%) & $19(76)$ & $9(75)$ & $11(84,6)$ & 0,82 \\
\hline Dislipidemia, n (\%) & $19(76)$ & $8(66,7)$ & $8(61,5)$ & 0,66 \\
\hline Tabagismo, n (\%) & $3(12)$ & $1(8,3)$ & $3(23,1)$ & 0,57 \\
\hline Infarto do miocárdio prévio, n (\%) & $5(20)$ & $7(58,3)$ & $8(61,5)$ & 0,01 \\
\hline
\end{tabular}


TABELA 2

Variáveis angiográficas e do procedimento

\begin{tabular}{|c|c|c|c|c|}
\hline & $\begin{array}{c}\text { SVB } \\
(n=25)\end{array}$ & $\begin{array}{c}\text { EES } \\
(n=12)\end{array}$ & $\begin{array}{c}\text { BES } \\
(n=13)\end{array}$ & Valor de $\mathrm{P}$ \\
\hline Vaso tratado, n (\%) & & & & 0,24 \\
\hline $\mathrm{DA}$ & $13(52)$ & $3(25,0)$ & $4(30,8)$ & \\
\hline $\mathrm{CX}$ & $4(16)$ & $5(41,7)$ & $6(46,2)$ & \\
\hline CD & $8(32)$ & $4(33,3)$ & $3(23,1)$ & \\
\hline Comprimento da lesão, mm & $11,7 \pm 4,0$ & $10,1 \pm 3,4$ & $12,9 \pm 5,9$ & 0,31 \\
\hline Diâmetro de referência, mm & $2,62 \pm 0,45$ & $2,73 \pm 0,36$ & $2,49 \pm 0,44$ & 0,34 \\
\hline \multicolumn{5}{|l|}{ Diâmetro da estenose, \% } \\
\hline Pré & $66,7 \pm 10,5$ & $67,0 \pm 9,2$ & $70,0 \pm 13,0$ & 0,79 \\
\hline Pós & $8,4+4,0$ & $8,2+4,2$ & $5,6+2,5$ & 0,58 \\
\hline \multicolumn{5}{|l|}{ Diâmetro luminal mínimo, mm } \\
\hline Pré & $0,87 \pm 0,32$ & $0,90 \pm 0,25$ & $0,78 \pm 0,44$ & 0,52 \\
\hline Pós & $2,39 \pm 0,31$ & $2,66 \pm 0,26$ & $2,69 \pm 0,45$ & 0,03 \\
\hline Ganho agudo, mm & $1,51+0,41$ & $1,76+0,28$ & $1,9+0,42$ & 0,02 \\
\hline Pressão máxima do balão na pós-dilatação, atm & $16,7 \pm 4,0$ & $15,0 \pm 7,1$ & $19,1 \pm 3,8$ & 0,03 \\
\hline Relação balão:artéria & $1,08 \pm 0,14$ & $1,05 \pm 0,11$ & $1,16 \pm 0,12$ & 0,06 \\
\hline
\end{tabular}

0,42 mm, $P=0,02)$. A pressão máxima de inflação do balão na pós-dilatação foi intermediária para o SVB $(16,7 \pm 4,0 \mathrm{~atm}$ vs. 15,0 \pm 7,1 atm vs. 19,1 $\pm 3,8 \mathrm{~atm}$; $P=0,03)$, assim como a relação balão:artéria $(1,08 \pm$ $0,14$ vs. $1,05 \pm 0,11$ vs. $1,16 \pm 0,12 ; \mathrm{P}=0,06)$.

\section{Avaliação da retração aguda dos stents}

Os parâmetros angiográficos relacionados à avaliação da retração aguda estão demonstrados na Tabela 3 . A retração aguda e o porcentual de retração aguda foram numericamente maiores no grupo tratado com SVB em relação aos indivíduos que receberam stents metálicos (EES e BES), porém tal diferença não atingiu significância estatística $(0,21 \pm 0,13 \mathrm{~mm}$ vs. 0,15 \pm $0,08 \mathrm{~mm}$ vs. $0,14 \pm 0,08 \mathrm{~mm} ; \mathrm{P}=0,21$; e $7,0 \pm 4,6 \%$ vs. $5,0 \pm 2,2 \%$ vs. $5,7 \pm 4,1 \%$; $P=0,16$, respectivamente, para SVB, EES e BES).

\section{Relação das variáveis angiográficas e do procedimento com o percentual de retração aguda dos stents}

Na Tabela 4, é demonstrada a relação das variáveis angiográficas e do procedimento com o porcentual de retração aguda. A relação balão:artéria $\geq 1,1$ apresentou maior porcentagem de retração aguda no grupo SVB $(P=0,05)$. As demais variáveis não se correlacionaram com a ocorrência de retração aguda dos stents.

\section{DISCUSSÃO}

O principal achado do estudo foi mostrar que o SVB ABSORB apresentou retração aguda um pouco maior em comparação aos dois stents metálicos de segunda geração, embora não significativa.

Um dos benefícios dos stents metálicos é fornecer suporte vascular adequado, prevenindo a oclusão aguda bem como as retrações aguda e tardia do vaso. Essa propriedade é necessária durante a fase de cicatrização do segmento tratado, tornando-se dispensável posteriormente. A presença permanente de um stent metálico pode interferir na motricidade e no remodelamento do vaso; enjaulá-lo de forma permanente, impedindo a revascularização cirúrgica nos casos em que são necessários longos segmentos tratados (full metal jacket); encarcerar ramos secundários; e prejudicar a realização de imagens não invasivas das artérias coronárias, como a angiotomografia e a ressonância nuclear magnética. ${ }^{2}$

Os SVB surgiram com a proposta de trazer suporte vascular transitório na fase crítica de cicatrização após a ICP e, ao mesmo tempo, modular a hiperplasia intimal reparadora, por meio da liberação dos fármacos antiproliferativos. Como as plataformas bioabsorvíveis são mais flexíveis que as metálicas, há preocupação se esses novos dispositivos forneceriam a mesma força radial que as plataformas metálicas. Estudos clínicos prévios, que avaliaram a taxa de retração aguda pós-implante de stents metálicos não farmacológicos, demonstraram variação entre 3 e 15\%. ${ }^{4-7}$ Essa ampla variação nas taxas de retração aguda foi atribuída, em parte, ao material e ao design do stent, e também à diferença nas definições de retração aguda. 
TABELA 3

Parâmetros angiográficos relacionados à retração aguda dos stents

\begin{tabular}{|c|c|c|c|c|}
\hline & $\begin{array}{c}\text { SVB } \\
(n=25)\end{array}$ & $\begin{array}{c}\text { EES } \\
(n=12)\end{array}$ & $\begin{array}{c}\text { BES } \\
(n=13)\end{array}$ & Valor de $\mathrm{P}$ \\
\hline Diâmetro do balão na pressão máxima de inflação do balão, mm & $2,82 \pm 0,32$ & $2,99 \pm 0,28$ & $3,09 \pm 0,42$ & 0,11 \\
\hline Diâmetro do stent após o esvaziamento do balão, mm & $2,60 \pm 0,31$ & $2,83 \pm 0,23$ & $2,95 \pm 0,41$ & 0,18 \\
\hline Retração aguda, mm & $0,21 \pm 0,13$ & $0,15 \pm 0,08$ & $0,14 \pm 0,08$ & 0,21 \\
\hline Retração aguda, \% & $7,0 \pm 4,6$ & $5,0 \pm 2,2$ & $5,7 \pm 4,1$ & 0,16 \\
\hline
\end{tabular}

TABELA 4

Relação das variáveis angiográficas e do procedimento com a porcentagem de retração aguda dos stents

\begin{tabular}{|c|c|c|c|c|c|c|c|}
\hline & \multicolumn{2}{|r|}{ SVB } & \multicolumn{2}{|r|}{ EES } & \multicolumn{2}{|r|}{ BES } & \multirow[b]{2}{*}{ Valor de $\mathrm{F}$} \\
\hline & $\mathbf{n}$ & Retração (\%) & $\mathbf{n}$ & Retração (\%) & $\mathbf{n}$ & Retração (\%) & \\
\hline \multicolumn{8}{|l|}{ DRV, mm } \\
\hline$\geq 3,0$ & 6 & $6,6 \pm 1,4$ & 3 & $5,2 \pm 3,9$ & 3 & $3,7 \pm 3,4$ & 0,61 \\
\hline$<3,0$ & 19 & $7,8 \pm 5,2$ & 9 & $4,9 \pm 1,3$ & 10 & $4,4 \pm 2,4$ & 0,21 \\
\hline Valor de P & & $>0,99$ & & 0,60 & & $>0,99$ & \\
\hline \multicolumn{8}{|c|}{ Pressão máxima, atm } \\
\hline$>16$ & 5 & $9,4 \pm 5,8$ & 7 & $5,1 \pm 2,7$ & 10 & $4,5 \pm 2,5$ & 0,51 \\
\hline$\leq 16$ & 20 & $7,0 \pm 4,2$ & 5 & $4,8 \pm 1,2$ & 3 & $3,2 \pm 2,8$ & 0,17 \\
\hline Valor de $\mathrm{P}$ & & 0,82 & & 0,88 & & 0,46 & \\
\hline \multicolumn{8}{|c|}{ Relação balão:artéria } \\
\hline$<1,1$ & 15 & $6,1 \pm 3,5$ & 8 & $4,9 \pm 2,5$ & 5 & $5,2 \pm 2,6$ & 0,76 \\
\hline Valor de P & & 0,09 & & 0,93 & & 0,35 & \\
\hline
\end{tabular}

A ideia de dispositivos de SVB não é nova, pois diversos tipos de modelos foram testados em estudos experimentais. O stent de Igaki-Tamai foi o primeiro dispositivo bioabsorvível testado em humanos no final da década de $1990 .{ }^{8}$ Sua plataforma, composta de PLLA, não possuía fármaco antiproliferativo em sua composição. Nesse trabalho pioneiro, a taxa de retração aguda foi de $22 \%$, usando metodologia diferente da empregada no presente estudo.

Estudos publicados mais recentemente que utilizaram a mesma metodologia, comparando o retração aguda do SVB com stents metálicos de segunda geração, demonstraram resultados semelhantes ao do nosso estudo. Tanimoto et al. ${ }^{9}$ compararam o SVB com o EES e constataram que o percentual de retração aguda foi de $6,9 \pm 7,0 \%$ no grupo SVB e $4,3 \pm 7,1 \%$ no grupo EES $(P=0,25)$. Onuma et al. ${ }^{10}$ compararam a retração aguda de duas versões do SVB ABSORB (revision 1.0 e revision 1.1) com o stent $X I E N C E^{\circledR} \vee$ e descobriram que a retração aguda do SVB 1.1 foi um pouco maior do que os EES metálicos (EES: 4,3 \pm 7,1\%; SVB 1.0: 6,9 \pm $7,0 \%$; SVB 1.1: 6,7 $\pm 6,4 \% ; P=0,22$ ). Recentemente, foram apresentados os dados de retração aguda de outro SVB, o DESolve, mostrando também boa força radial e retração aguda de $6,4 \pm 4,6 \% .{ }^{11}$ Um terceiro SVB testado foi o DREAMS ${ }^{\circledR}$ (Biotronik, Bülach, Suíça), cuja estrutura é composta por magnésio, e o fármaco antiproliferativo é o paclitaxel, avaliado no estudo firstin-man BIOSOLVE-I, demonstrando bom desempenho em relação à retração aguda $(9,19 \pm 7,23 \%){ }^{12}$

Uma vez que a retração aguda é resultante do equilíbrio entre o recuo elástico da parede vascular e a força radial exercida pelo stent, ela pode ser alterada por características da lesão ou do próprio procedimento, como o diâmetro de referência do vaso, a pressão máxima de inflação do balão e o superdimensionamento do stent utilizado. Em relação ao diâmetro do vaso e a pressão máxima de inflação do balão, não houve diferença nas taxas de retração entre os três grupos. Porém, no grupo de pacientes que apresentou relação balão:artéria $\geq 1,1$, houve maior taxa de retração no grupo SVB $(\mathrm{P}=0,05)$. Esses resultados ressaltam a importância de dimensionar corretamente o vaso e, possivelmente, preparar a lesão, antes do implante do SVB. 
O presente estudo teve algumas limitações, pois foi realizado em um único centro, não randomizado, e com um pequeno número de pacientes. Além disso, os resultados podem também estar relacionados à inclusão na análise de lesões de baixa complexidade.

\section{CONCLUSÕES}

Nesta casuística, o suporte vascular bioabsorvível ABSORB demonstrou boa força radial, com retração aguda ligeiramente maior, embora não significativa, em comparação aos stents metálicos de segunda geração. Estudos com maior número de pacientes e em cenários mais complexos são necessários para confirmar essas observações preliminares.

\section{CONFLITO DE INTERESSES}

Os autores declaram não haver conflito de interesses relacionado a este manuscrito.

\section{REFERÊNCIAS}

1. Brophy JM, Belisle P, Joseph L. Evidence for use of coronary stents: a hierarchical bayesian meta-analysis. Ann Intern Med. 2003;138(10):777-86.

2. Farb A, Weber DK, Kolodgie FD, Burke AP, Virmani R. Morphological predictors of restenosis after coronary stenting in humans. Circulation. 2002;105(25):2974-80.

3. Bourantas CV, Zhang Y, Farooq V, Garcia-Garcia HM, Onuma $Y$, Serrys PW. Bioresorbable scaffolds: current evidence and ongoing clinical trials. Curr Cardiol Rep. 2012;14(5):626-34.

4. Haude M, Erbel R, Issa H, Meyer J. Quantitative analysis of elastic recoil after balloon angioplasty and after intracoronary implantation of balloon-expandable Palmaz-Schatz stents. J Am Coll Cardiol. 1993;21(1):26-34.
5. Fischman DL, Leon MB, Baim DS, Schatz RA, Savage MP Penn I, et al. A randomized comparison of coronary-stent placement and bal- loon angioplasty in the treatment of coronary artery disease. Stent Restenosis Study Investigators. N Engl J Med. 1994;331(8):496-501.

6. Rechavia E, Litvack F, Macko G, Eigler NL. Influence of expanded balloon diameter on Palmaz-Schatz stent recoil. Cathet Cardiovasc Diagn. 1995;36(1):11-6.

7. Bermejo J, Botas J, Garcia E, Elizaga J, Osende J, Soriano J, et al. Mechanisms of residual lumen stenosis after high-pressure stent implantation: a quantitative coronary angiography and intravascular ultrasound study. Circulation. 1998;98(2):112-8.

8. Tamai H, Igaki K, Kyo E, Kosuga K, Kawashima A, Matsui S, et al. Initial and 6-month results of biodegradable poly-L-lactic acid coronary stents in humans. Circulation. 2000;102(4): 399-404.

9. Tanimoto S, Serruys PW, Thuesen L, Dudek D, de Bruyne B, Chevalier B, et al. Comparison of in vivo acute stent recoil between the bioabsorbable everolimus-eluting coronary stent and the everolimus-eluting cobalt chromium coronary stent: insights from the ABSORB and SPIRIT trials. Catheter Cardiovasc Interv. 2007;70(4):515-23.

10. Onuma Y, Serruys PW, Gomez J, Bruyne B, Dudek D, Thuesen $\mathrm{L}$, et al. Comparison of in vivo acute stent recoil between the bioresorbable everolimus-eluting coronary scaffolds (revision 1.0 and 1.1) and the metallic everolimus-eluting stent. Catheter Cardiovasc Interv. 2011;78(1):3-12

11. Costa RA, Abizaid A, Webster M, Stewart J, Costa JR, Lima M, et al. Acute scaffold recoil with the novel myolimus-eluting bioresorbable vascular scaffold in the treatment of de novo, non-complex coronary lesions [Poster apresentado no Congresso SBHCl/SOLACI, São Paulo, 2013].

12. Haude M, Erbel R, Erne P, Verheye S, Degen H, Böse D, et al. Safety and performance of the drug-eluting absorbable metal scaffold (DREAMS) in patients with de-novo coronary lesions: 12 month results of the prospective, multicentre, first-in-man BIOSOLVE-I trial. Lancet. 2013;381(9869):836-44. 